\title{
Quality of life in patients undergoing surveillance for non-muscle invasive bladder cancer-a systematic review
}

\author{
Arvind Nayak ${ }^{1}$, Joanne Cresswell ${ }^{2}$, Paramananthan Mariappan ${ }^{3,4}$ \\ ${ }^{1}$ RCS Robotic Fellow Urology, Lister Hospital, Stevenage, Coreys Mill Lane, Stevenage, UK; ${ }^{2}$ Department of Urology, James Cook University \\ Hospital, Middlesbrough, UK; ${ }^{3}$ Department of Urology, University of Edinburgh, Edinburgh, UK; ${ }^{4}$ Department of Urology, Western General \\ Hospital, Crewe Road South, Edinburgh, UK \\ Contributions: (I) Conception and design: P Mariappan, J Cresswell; (II) Administrative support: J Cresswell; (III) Provision of study materials or \\ patients: A Nayak; (IV) Collection and assembly of data: A Nayak; (V) Data analysis and interpretation: A Nayak; (VI) Manuscript writing: All \\ authors; (VII) Final approval of manuscript: All authors. \\ Correspondence to: Arvind Nayak, MBBS, MS, McH (Urol), FRCS (Urol). RCS Robotic Fellow Urology, Lister Hospital, Stevenage, Coreys Mill \\ Lane, Stevenage, SG1 4AB, UK. Email: arvindnayakk@gmail.com.
}

\begin{abstract}
Background: The main objective of this study was to evaluate the various instruments available to evaluate the quality of life $(\mathrm{QoL})$ in patients diagnosed with non-muscle invasive bladder cancer (NMIBC) undergoing surveillance.

Methods: A PubMed literature review was carried out with query terms ("Urinary Bladder Neoplasms" [Mesh] OR "Bladder malignancy") AND ("quality of life") including all studies up to June 2020. This resulted in 576 peer-reviewed articles. A further 12 articles from additional sources were included. A total of 473 articles were eliminated due to lack of relevance to the topic of concern. A further 93 articles evaluating NMIBC and articles evaluating Radiotherapy were excluded and a total of 22 studies were studied.

Results: In total, 22 studies were identified. The vast majority of studies were prospective descriptive studies ( $\mathrm{n}=9$ ), while there were 7 cross-sectional surveys and 6 randomised controlled trials. Most studies evaluated the impact of intravesical treatment on QoL. NMIBC survivors had significantly lower QoL compared to the general population, Surveillance strategies involving repeated intravesical therapies and cystoscopies have a negative impact on QoL with impaired physical function and mental health.

Conclusions: This article emphasizes the importance of assessing the QoL in patients with NMIBC undergoing long term surveillance, as they represent the majority of bladder cancer patients. Development and validation of specific instruments to measure QoL in patients with NMIBC are desperately needed to assess, better understand, and manage the burden of disease and healthcare in this group of patients.
\end{abstract}

Keywords: Quality of life (QoL); bladder cancer; non-muscle invasive bladder cancer (NMIBC); surveillance

Submitted Oct 11, 2020. Accepted for publication Feb 04, 2021.

doi: $10.21037 /$ tau-20-1333

View this article at: http://dx.doi.org/10.21037/tau-20-1333

\section{Introduction}

Bladder cancer (BC) is the $11^{\text {th }}$ commonest cancer in the UK, accounting for $3 \%$ of all new cancer cases, ranking $7^{\text {th }}$ in cancer incidence worldwide $(1,2)$. Men are more likely to be affected by BC compared to women-estimated as $73 \%$ and $27 \%$ of cases in the UK respectively (2). Figures from 2010 revealed the total number of bladder cancer survivors in the UK was 69,100 (2). The cost of the management of bladder cancer from diagnosis to mortality is the highest among all cancers (3).

Urothelial carcinoma is the most frequent histologic variant of BC, accounting for $90 \%$ of cases. The depth of cancer invasion is used to categorize $\mathrm{BC}$ into non-muscle invasive BC (NMIBC) and muscle invasive BC (MIBC). 
Approximately $75 \%$ of new bladder cancer diagnoses are NMIBC (4). The initial treatment for NMIBC is Transurethral Resection of a Bladder Tumour (TURBT) followed by periodic cystoscopic surveillance and selective intravesical therapy (5). The recurrence rate of NMIBC is between $60-70 \%$ with progression to MIBC occurring in $20-30 \%$ (6). The high 5 -year survival for NMBIC of around $80 \%$ (7) is responsible for its high prevalence. Thus, patients undergo follow-up (often life-long) with repeated radiological imaging, endoscopic inspection, biopsies, tumour resections and frequent (in selected patients) intravesical treatments.

Although the prevalence of NMIBC is high and treatment burden in prolonged and intensive, relatively little has been explored with regards to the impact of the disease/treatment on the patient's quality of life (QoL). Most of the studies concerning QoL have focussed on the effect of radical cystectomy and various urinary diversion methods (8). Population based studies in BC patients have revealed both statistically and clinically significant decline in physical health-related quality of life (HR-QoL), irrespective of stage $(9,10)$. Determining the impact on health status and QoL in patients with NMIBC is crucial given its high prevalence, protracted course of management and high recurrence rate with consequent repeat cycles of treatment. This article aims to explore QoL in patients undergoing surveillance following a diagnosis of NMIBC. We present the following article in accordance with the PRISMA reporting checklist (available at http://dx.doi. org/10.21037/tau-20-1333).

\section{Methods}

\section{Evidence acquisition}

A comprehensive search of published literature until June 2020 was carried out with query terms ("Urinary Bladder Neoplasms" [Mesh] OR "Bladder malignancy") AND ("quality of life"). This resulted in 576 peer-reviewed articles (Figure 1). Pertinent references from articles which were analysing QoL in NMIBC were also included. 473 articles were eliminated due to lack of relevance to the topic of concern. The focus of this paper is NMIBC, hence literature on muscle invasive and metastatic diseases were excluded. In total, 22 studies (Table 1) were identified and included in this review. As seen in Table 1, the vast majority of studies were prospective descriptive studies $(n=9)$, while there were 7 cross-sectional surveys and 6 randomised controlled trials. Most studies evaluated the impact of intravesical treatment on QoL.

\section{Data extraction}

Titles and/or abstracts of studies yielded by search strategy and from additional sources (i.e., references from relevant papers) were screened independently. Studies were evaluated using the Prisma checklist (33). A narrative review for QoL in patients with NMIBC was composed. No statistical analysis was required due to the small number of studies.

\section{Results}

\section{QoL instruments}

HR-QoL and symptom burden are central concerns for patients and clinicians, hence patient-reported outcomes (PROs) can be useful tools for treatment assessment and to guide the formulation of recommendations for NMIBC. Studies and clinical experience have shown that depending on the modality of treatment adopted, the adverse effects and toxicities experienced by patients vary. Comparative analysis among the modes of treatment such as chemotherapy $v s$. BCG, induction $v s$. maintenance therapy and the impact of single instillation chemotherapy, have also emphasized the severe and persistent effects these can have on HR-QoL.

The QoL instruments used in the different studies were varied (Table 2) and can be categorised as generic, bladder specific and symptom specific tools. Several combinations were also noted. Among the generic questionnaires, EORTC QLQ Core 30, was the most frequently used questionnaire - used in 6 articles $(11,13,14,23,24,28)$. On the other hand, the Medical Outcomes Study 36-item Short Form was used in 4 articles $(15,16,30,34)$; the Veterans RAND 12-item health survey in 2 studies $(16,34)$; the FACT-G in one study (21) and the visual analogue scale evaluating general wellbeing was used in one study (29).

Bladder specific instruments such as the bladder cancer index (BCI) was used in 2 studies $(15,22)$; the EORTC QLQY Bladder Cancer Superficial 24 (EORTC QLQBLS24; renamed as EORTC-QLQ NMIBC24) in 2 studies $(23,25)$ while the FACT-BC was used in 2 articles $(12,21)$.

Symptom specific tools assessed Urinary issues $(15,24,35)$; sexual health $(15,19,22,25-27,29)$ and psychological health 


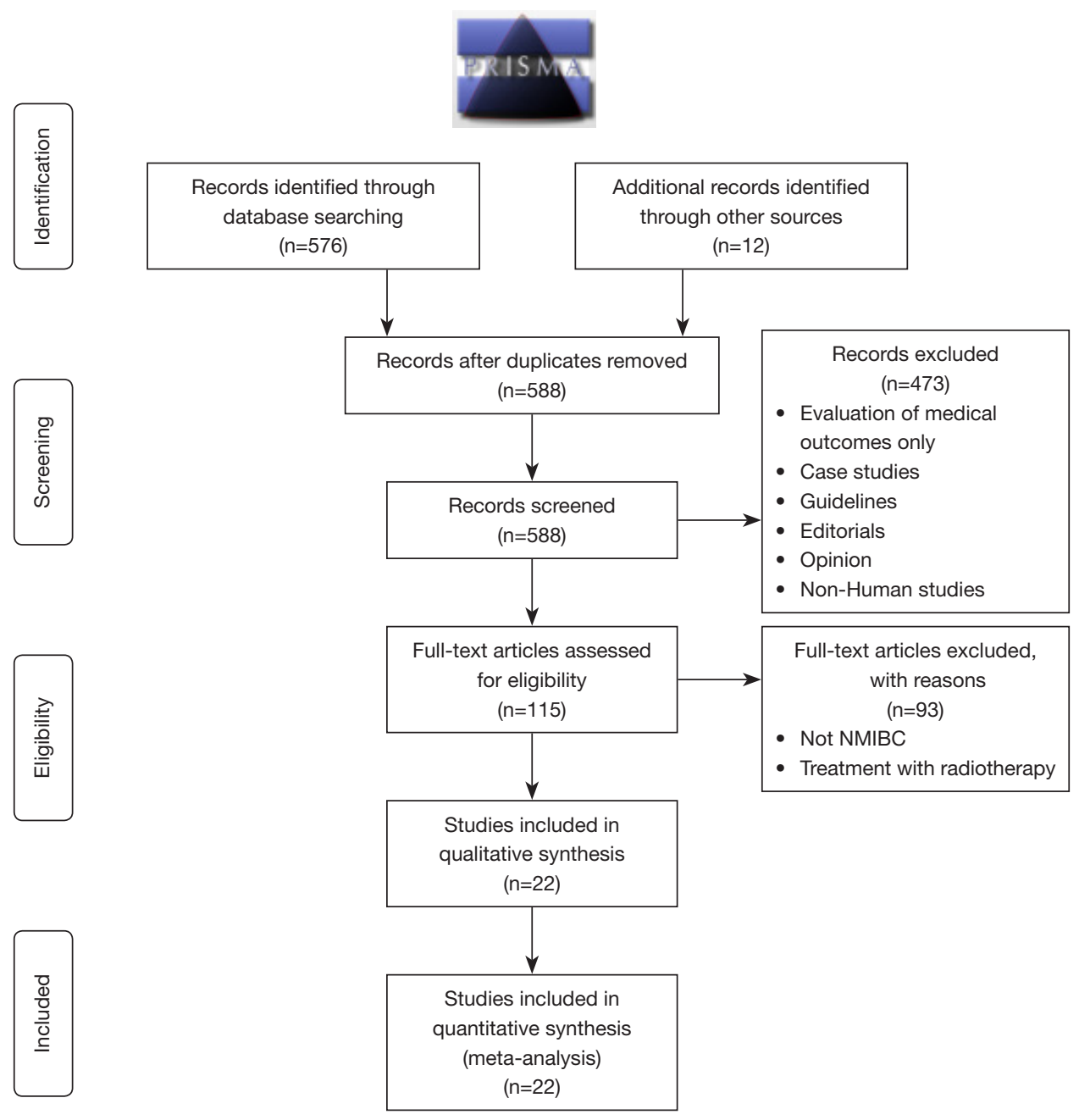

Figure 1 PRISMA 2009 flow diagram.

$(14,17,21-23,26,32)$ using a variety of tools as noted in Table 2.

Some tools have been translated to other languages and subsequently validated-Park et al. (13), using a rigorous process, developed and validated a Korean version of the EORTC-QLQ C30 and NMIBC24 questionnaires.

\section{Impact of NMIBC (and treatment) on physical QoL}

In addition to the symptoms related to the cancer per se, patients with NMIBC also suffer from physical symptoms resulting from repeated treatments such as TURBT, intravesical treatments and surveillance procedures such as check cystoscopies; which can have an impact on the QoL. In a study by Brisbane et al., using the Surveillance,
Epidemiology, and End Results-Medicare Health Outcome Survey (SEER-MHOS) database, there was a significant decline in the physical component of HR-QoL when compared with non-cancer controls - on sub-analysis, the significant decline in the physical component appeared to be limited to patients with high-risk NMIBC (16).

The impact of TURBT on the QoL has been assessed in a prospective study in Japan (30) which found that the QoL (measured as General Health Related QoL) was least at the third TURBT, but reached normalcy with 4 or more TURBTs. Patients' mental health was found to be worst at the initial TURBT (28). Conversely, Park and colleagues reported an overall improvement in urinary symptoms between baseline and post-TURBT visits (13). This may be attributable to the fact that bladder cancer might have 
Table 1 Table summarizing study characteristics

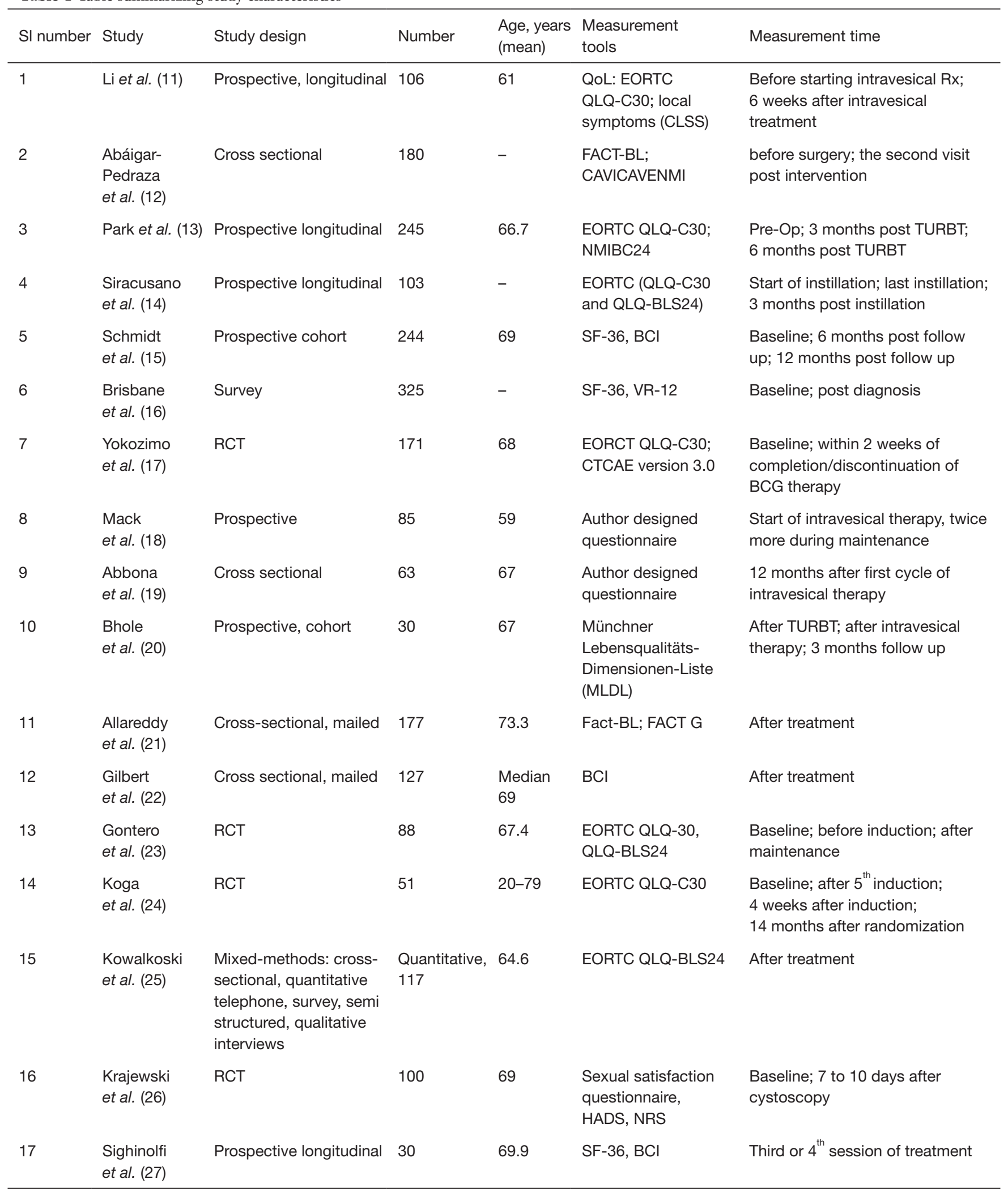

Table 1 (continued) 
Table 1 (continued)

\begin{tabular}{|c|c|c|c|c|c|c|}
\hline SI number & Study & Study design & Number & $\begin{array}{l}\text { Age, years } \\
\text { (mean) }\end{array}$ & $\begin{array}{l}\text { Measurement } \\
\text { tools }\end{array}$ & Measurement time \\
\hline 18 & $\begin{array}{l}\text { Singer } \\
\text { et al. (28) }\end{array}$ & Cross sectional & 210 & 67 & EORTC-QLQ & 30-40 days after last treatment \\
\hline 19 & $\begin{array}{l}\text { van der Aa } \\
\text { et al. (29) }\end{array}$ & Cross sectional & 142 & 65 & $\begin{array}{l}\text { VAS, EORTC-QLQ- } \\
\text { BLS24 }\end{array}$ & $\begin{array}{l}\text { The start of surveillance; } \\
<3 \text { months after the diagnosis of } \\
\text { primary or recurrent NMIBC }\end{array}$ \\
\hline 21 & Cox et al. (31) & $\mathrm{RCT}$ & 472 & 65.9 & EQ-5D & $\begin{array}{l}\text { Baseline; 12, 24, 36-month } \\
\text { follow up }\end{array}$ \\
\hline
\end{tabular}

Table 2 The QoL questionnaires used

Cancer specific QoL questionnaires

- Generic

- Medical Outcomes Study 36-item Short Form

o Veterans RAND 12-item health survey

- ED-5Q

- Cancer specific

- European Organization for Research and Treatment of Cancer Quality of Life Questionnaire [EORTC QLQ] Core 30

- Functional Assessment of Cancer Therapy [FACT]-General

Disease specific QoL questionnaires

- FACT-bladder cancer

- $\mathrm{BCl}$

- EORTC QLQ-Bladder Cancer Superficial 24 (EORTC QLQBLS24); renamed EORTC-QLQ NMIBC24

Symptom specific QoL questionnaires

- Physical symptoms

- Core Lower Urinary Tract Symptom Score

- International Prostate Symptom Score

○ Numeric Rating Scale

- Psychological symptoms

o Brief Symptom Index-18

- Illness Intrusive Rating Scale

o Hospital Anxiety and Depression Scale

- Sexual symptoms

- Sexual Satisfaction Questionnaire

o 5-item International Index of Erectile Function 
caused some of the pre-TURBT urinary symptoms.

The impact of intravesical therapy on QoL was analysed in several studies. The findings of Li et al. (11) revealed significant decrease in QoL compared to the baseline, owing to the increase in symptoms (pain, frequency and diarrhoea) at the $6^{\text {th }}$ week post intravesical treatment review. The lower urinary tract symptoms (LUTS) showed significant negative associations with global health status and were positively associated with pain (11). A prospective study from Korea found worsening of LUTS in about $40 \%$ of the patients (13). An Italian study reported the rates of side effects of treatment to be $46.6 \%$ during the last instillation of induction course (with both BCG and Mitomycin C) and $47.5 \%$ at 3 months following the last induction instillation-there appeared to be a commensurate drop in QoL following the induction course, which then returned to the baseline within 3 months (14). These findings were more evident in the patients aged 70 years and above, and particularly more in those who received BCG.

A randomized controlled trial compared patients with NMIBC post BCG treatment in maintenance therapy and observation only groups (24). Although during the induction therapy, the symptom burden was high, at $70 \%$ mainly including bladder-related side effects such as dysuria $(82.2 \%)$, day time frequency $(82.2 \%)$, and visible haematuria $(72.2 \%)$ these did not reflect in the QoL scores in long term. Mack and Frick (18), evaluated patients using an author designed questionnaire and reported $83 \%$ of the patients to have symptoms during induction of BCG with slightly lower proportion during monthly maintenance therapy-the overall QoL seemed to improve into the maintenance phase of treatment. From these studies we can infer that QoL is independent of symptom development. This lack of association, makes validation of QoL instruments challenging and complicated

The effects of specific intravesical agents on the QoL have also been evaluated. A prospective randomized study (23) showed that intravesical gemcitabine when compared to group receiving intravesical BCG had fewer urinary symptoms on univariate analyses. However multivariate analyses concluded that the differences were not significant in all the dimensions of QoL. In addition, the duration of treatment was not significantly different in both the groups and physical functioning gradually deteriorated in both groups alike. The HYMN study, a randomised controlled trial comparing Hyperthermic Mitomycin versus $2^{\text {nd }}$ course of BCG for patients with intermediate or high risk recurrence following an induction (or induction and maintenance) course of BCG demonstrated no difference in HR-QoL between the two treatment arms, although patients treated with Hyperthermic intravesical therapy, rated their health status higher than controls at 3, 6, and 9 months of follow-up (32).

\section{The impact on diagnosis of recurrence}

A randomised trial comparing celecoxib versus standard treatment (BOXIT study) of 472 patients diagnosed with recurrent NMIBC, reported that patients diagnosed with recurrence experience decrements in HR-QoL (31). This study demonstrated that patients with high grade recurrence and progression were associated with statistically significant HR-QoL decrements. In contrast, patients with low grade recurrences had positive but statistically insignificant increment in QoL compared with patients without cancer. Secondary analysis evaluating timing of recurrence with QoL showed that, patients with high grade NMIBC recurrence in the first-year experienced larger decrements in QoL compared with those with recurrence in subsequent years. Patients undergoing radical cystectomy for either progression or high-grade recurrence was associated with a decrement in HR-QoL. From this trial, the authors suggest that not all cases of NMBIC recurrence should be considered equal with respect to the effects on patient QoL

\section{Impact on psychological QoL}

In addition to the effect on physical health, both cancer diagnosis and its treatment have implications on the psychological health of patients. Singer et al. (28) demonstrated that the emotional functioning of patients with BC was lower than general population. The Mental health scores were found to significantly deteriorate at the time of diagnosis (34).

The most severe deterioration in Mental health scores were at the initial TURBT and reached normalcy with subsequent TURBT (30).

Intravesical therapy also tends to have an impact on the psychological aspect of life. Abbona et al. demonstrated Intravesical therapy altered self-esteem (38\% of respondents) (19). The impact of induction and maintenance intravesical therapy on the psychological state of mind was studied by Koga et al. (24). They demonstrated that both induction and maintenance therapy with BCG did not negatively affect the QoL, thereby the emotional functioning scale improved significantly. Patients' 
acceptance of their disease state and their compliance with both induction and maintenance therapy using BCG contributed to improvement in their QoL. The type of intravesical agent used may also have an impact on the mental state of the patient. Emotional functioning in the gemcitabine group improved over time, but that in the BCG group decreased (23).

The patient with NMIBC tend to have regular cystoscopies as a part of surveillance which can impact the mental state. Krajewski et al. demonstrated anxiety was significantly decreased after flexible cystoscopy (26). Mack and Fick reported that patients undergoing cystoscopy experienced comparable symptoms to those undergoing intravesical therapy (18).

\section{Impact on sexual bealth}

The diagnosis of bladder cancer has an impact on sexual health and hence QoL. In a study by Kowalkowski $e t$ al., among sexually active participants, $50 \%$ reported sexual dysfunction encompassing-erectile dysfunction (60\%), vaginal dryness $(62.5 \%)$, ejaculatory dysfunction $(43.1 \%)$; $23.2 \%$ reported worry about contaminating partner with treatment agents and $38.8 \%$ sexual inactivity. Negative impacts on their relationships were reported. More women were significantly inactive compared to men. Interest in sexual activity appeared to be significantly different according to the time since diagnosis-groups categorised as "not interested in sex" (at 16.7 months) shifted to "at least some interest in sex" (at 25.4 months) (25).

With regards to TURBT and its impact on sexual health, Schmidt et al. demonstrated that sexual function appeared to reduce in patients with NMIBC in the first year following TURBT with/without intravesical treatment (15).

Intravesical therapy also tends to have an impact on sexual wellbeing of the patient. All the male patients were found to have impaired sexual function after intravesical BCG in a prospective study. A decrease in erectile function was reported within 2-3 days of the instillation and gradual recovery of function was observed at 1-month follow-up after the final instillation (27). Mack and Frick reported that sexual activity was not effected greatly during induction phase, however an improvement was noted during maintenance therapy (18).

Patients undergoing surveillance by cystoscopy and their impact on sexual health was studied by Krajewski et al. (26)sexual satisfaction was significantly decreased after rigid cystoscopy but not in those undergoing flexible cystoscopy.

\section{Discussion}

NMIBC can be considered a chronic disease, involving frequent relapses after the initial treatment. Close surveillance is therefore needed and adjuvant treatment aimed at bladder preservation may be required. The benefits of such treatments may accrue only after an extended period of follow-up; however, substantial subjective psychological, physical, and local toxic effects of topical therapy are reported earlier. In the past, the pivotal data used to assess the success of bladder cancer management were overall survival, tumour free survival and recurrence-free rates. The reviews from early 2000s suggested that QoL assessments in clinical studies were somewhat heterogenous in relation to the tools utilised; the use of non-validated tools; lacking assessment of the impact of recurrence and progression on QoL; did not account for confounders in the assessment and usually had a short duration of follow up (36-38). Reassuringly, there has been a shift in recent times, to incorporate the data on QoL in patients with bladder cancer during treatment (surgical and intravesical instillations) and surveillance, while evaluating the impact of recurrence and progression (29). The International Bladder Cancer Group have also recommended, recently, that HRQoL should be included as secondary endpoints in clinical trials (39). The caveat, of course, is that the impact on QoL in the highly selective patients recruited into clinical trials might not be similar to that seen in the real world (i.e., lacking external validity). More recently, review articles have looked at the various tools to assess QoL in NMIBC and have systematically looked at impact of TURBT, intravesical therapy, surveillance and impact of recurrence and progression on QoL $(40,41)$.

The standard initial step in the diagnosis and management is TURBT, including the resection of underlying detrusor muscle [with TaG1/low-grade (LG) tumours as exceptions] (42) as the absence of detrusor muscle in the specimen poses higher risk of residual disease (34) (up to $55 \%$ in T1 tumours) (43), early recurrence, under staging of the tumour (44) and poorer survival (45). This is, nonetheless, an invasive procedure with potential side effects and complications, all of which can adversely affect patients' QoL.

Based on the biological potential of the cancer, guidelines recommend postoperative intravesical chemotherapy or BCG therapy (which includes both induction and maintenance treatment) to reduce the risk of recurrence and potentially, progression. And should there be a progression in the disease despite the instillations early recourse to 
cystectomy is advised (46). Therefore, not only can the need for and side effects of the instillations impact the QoL, the fear that treatment might escalate to radical intervention can instil a sense of constantly "living on edge", further impacting the QoL.

Surveillance is also tailored according to the risk strata. The frequency of surveillance increases with the increase in risk accordingly impacting the QoL-most guidelines advocate 3-month cystoscopy for 2 years, then every 6 months for 5 years and then annually (39), provided there is no recurrence. Any recurrence that does not result in an escalation to radical treatment results in going back to more frequent surveillance with cystoscopies and upper tract imaging (39).

Complete removal of the tumour is of paramount importance in the management of patients with NMIBC, so as to reduce progression to muscle invasion, recurrence or distant metastasis (47) and ensure that the benefits of treatment outweigh the side effects. It is essential to have knowledge of the different adverse reactions associated with the various treatment options such as chemotherapy $v s$. BCG, induction $v s$. maintenance therapy, impact of single instillation chemotherapy and their resultant effects on HRQoL $(48,49)$.

In the studies we reviewed, the authors have often chosen the QoL tools depending upon the aspect they wanted to address in their study, i.e., whether it is health concerns only or cost effectiveness of surveillance. Choice of the tool also depends on the available resources for the study. The studies on QoL have impacted clinical practice as they have highlighted the importance of patient education regarding the disease, it's treatment options and their possible outcomes beyond simply cancer recurrence or progression. The studies have also emphasised the importance of assessing the QoL by the oncology healthcare team as a routine, with the aim to improving it.

QoL is a multidimensional concept that involves physical as well as psychosocial health (50). Various instruments have been used to measure QoL. They can be broadly divided into Generic, Bladder cancer specific and non-QoL symptom scores. Generic QoL instruments like EQ-5D$5 \mathrm{~L} / 3 \mathrm{~L}, \mathrm{SF} 36$ aim to target a wider demographic and include broader domains-the domains measured in EQ-5D-5L include mobility, self-care, usual activities, pain/discomfort, anxiety/depression. These tools are designed to cater to wider range of health conditions making them less sensitive in picking up cancer related QoL aspects. These tools also fail to cover specific cancer treatment and outcome details. Cancer-specific instruments like FACT-G, EORTC QLQ C30 are intended to target a specific population and may allow comparative analyses across studies. FACT-G incorporates 27 items spanning 4 domains - functional, physical, social, and emotional. The EQ-5D-5L is used to calculate quality-adjusted life years (QALYs), which are composite outcomes of survival and HR-QoL. It consists of 5 domains ( 3 or 5 response levels) including mobility, self-care, regular activities, pain, anxiety/depression. These cancer specific questionnaires failed to cover skin problems, systemic side effects of treatment (BCG) such as joint pain and stiffness.

Of late, bladder cancer specific instruments have been designed; however, they are yet to be tested and validated. The FACT-Bladder Cancer (FACT-BL) (51) instrument is a conglomerate of FACT-general version (FACT-G) and 12 other items like incontinence, diarrhoea, body image, sexual function and stoma care which are specific to bladder cancer. The BCI instrument holds good for all patients with bladder cancer, irrespective of tumour course or treatment (35). It assesses 36 items spanning domains of urinary, bowel, and sexual symptoms.

The EORTC- QLQ-C30 questionnaire although a cancer specific questionnaire did not cover specific aspects of NMIBC, therefore a 24-item questionnaire (EORTC QLQ-NMIBC24) for patients with NMIBC (Ta, T1, carcinoma in situ) (52) was developed by EORTC, containing items related to adversities of intravesical treatment. These two tools when used together represented $68 \%$ of content important in NMIBC (40).

Questionnaires focussing on specific symptoms include questionnaires such as IPSS for Lower urinary tract symptoms, IIEF for sexual function and various psychology questionnaires which are not specific to cancer and its treatment. Studies have also shown that symptoms do not always correlate with the QoL (18).

Not all of the questionnaires mentioned above are validated, a finding corroborated by the previous systematic review by Rutherford and colleagues (40). The BCI and FACT-Vanderbilt Cystectomy Index (FACTVCI) are the only validated $\mathrm{QoL}$ questionnaires specific to bladder cancer to date, however they are not specific to NMIBC (8). The studies for validity and reliability of EORTC QLQ-BLS24 and FACT Bladder Cancer instruments are ongoing (41). Thus, the common limitation in all the existing literature has been the lack of a validated bladder cancer diseasespecific instrument for assessing impact on QoL. One of the factors posing a challenge in validation of QoL tools is 
appearance of symptoms without impacting the QoL, this could be because perception of symptoms by individuals is subjective.

Research on psychosocial QoL and interventions to improve them are sparse, despite the considerable burden of mental health and social problems faced by NMIBC survivors. Further support and research are warranted to develop and validate bladder cancer specific QoL instruments to evaluate patients with NMIBC.

This review emphasizes the importance of designing and choosing QoL tools that are specific to NMIBC to overcome the problems associated with generic tools like inclusion of features that are irrelevant to the disease and omitting important information concerning the disease and its treatment. From this review, we also understand that QoL decline is most severe after the first few TURBT's and intravesical instillations and gradually improves with time.

Thus, in everyday practice, these tools not only serve as surveys but can be used to counsel patients about the impact of the NMIBC on QoL before starting therapy and provide personalized resources like patient information leaflets, support groups, links to website for the symptoms and concerns at every clinic visit. Such a practice would amount to a holistic approach, addressing the patient as a whole rather than just the cancer treatment. Educating the patient will also enable them to actively participate in decision making regarding the course of their management and make a trade-off between burden of surveillance to be cancer free and better QoL.

MDTs and tumour boards have made decision-making process and execution of plans in cancer management more streamlined. A key aspect which is made evident by this review is that the need of the hour is, to achieve a treatment strategy that balances the oncological concerns and QoL aspects in every patient. Addressing QoL details at MDT while planning the treatment would make it more appropriate rather than as a troubleshooting measure posttreatment.

This review reiterates the need for a paradigm shift in the management of cancer. Rather than focussing on merely adding number of years to life and bench-marking these measures, like 5-year survival rates as cornerstones of success, keeping QoL also as a central aim would bring about a change in interventions. Accordingly, surgeon reported outcome measures should incorporate QoL details alongside oncological results. Several measures to reduce treatment burden can have a positive effect QoL. This can include reducing the dose or frequency of BCG instillations. In the CUETO study, no overall difference in efficacy was noted in patients receiving one-third dose to full-dose BCG (53). A decrease in efficacy was noted with a reduction to one-sixth dose with no decrease in toxicity (54). Some studies have looked at reducing the frequency of intravesical therapy, however optimal number of induction instillations and the optimal frequency and duration of maintenance instillations are not fully known (6). Other studies have looked to reduce the burden of cystoscopy particularly in low risk patients as per NICE guidance (55) which may have a beneficial effect on QoL. Some studies have looked at omission of TURBT and treating low risk patients with chemoablation comprising of four once-weekly MMC 40-mg intravesical instillations. This may be particularly beneficial in elderly frail patients although no difference in QoL was noted when compared to patients undergoing surgery (56).

Current guidelines by EAU and NICE do not have specific recommendations regarding the measurement of QoL in patients with NMIBC. Accordingly, QoL aspects haven't been taken into consideration while postulating management protocols. In the future the committees formulating the guidelines could look into impact of management aspects on QoL for instance in those with intermediate-risk disease options of either offering prophylactic intravesical therapy to prevent recurrence whereby the oncological outcomes are the focus of the approach or the option to carry out regular cystoscopies and treat as and when a recurrence is detected, in a "cross the bridge when we get to it" approach which could mean better QoL for the patient. Intravesical therapies come with their own set of drawbacks and bearings on QoL and may amount to over caution in an intermediate risk NMIBC, therefore only cystoscopy surveillance could be an alternative. A background knowledge of implications of either option on the QoL would guide the patient and health care team to opt the route which is most suitable for the patient in terms of their QoL.

The step further in making the surveillance policy more patient- friendly are Urinary biomarkers and imaging which can be used as alternatives to cystoscopy in patients undergoing surveillance. Several biomarkers have been researched, however none are sufficiently effective when used in isolation (57). Further research in this field can circumvent the need for invasive surveillance and thereby improve the QoL. 


\section{Conclusions}

This review emphasizes the importance of assessing the QoL in patients with NMIBC undergoing long term surveillance, as they represent the majority of bladder cancer patients. It is important to note that despite the high prevalence of NMIBC and the protracted course of management and surveillance involved, studies addressing the QoL in the survivors are limited. We emphasize the importance of educating the patients and healthcare professionals about the disease, its treatment and surveillance as well as the impact on physical, psychosocial, and sexual health. Development and validation of specific instruments to measure QoL in patients with NMIBC are desperately needed to assess, better understand and manage the burden of disease and healthcare in this group of patients.

\section{Acknowledgments}

Funding: None.

\section{Footnote}

Provenance and Peer Review: This article was commissioned by the Guest Editors (Mieke Van Hemelrijck and Netty Kinsella) for the series "Expectant Management in Genitourinary Malignancies (Prostate, Bladder, Kidney)" published in Translational Andrology and Urology. The article has undergone external peer review.

Reporting Checklist: The authors have completed the PRISMA reporting checklist. Available at http://dx.doi. org/10.21037/tau-20-1333

Conflicts of Interest: The authors have completed the ICMJE uniform disclosure form (available at http:// dx.doi.org/10.21037/tau-20-1333). The series "Expectant Management in Genitourinary Malignancies (Prostate, Bladder, Kidney)" was commissioned by the editorial office without any funding or sponsorship. The authors have no other conflicts of interest to declare.

Ethical Statement: The authors are accountable for all aspects of the work in ensuring that questions related to the accuracy or integrity of any part of the work are appropriately investigated and resolved.
Open Access Statement: This is an Open Access article distributed in accordance with the Creative Commons Attribution-NonCommercial-NoDerivs 4.0 International License (CC BY-NC-ND 4.0), which permits the noncommercial replication and distribution of the article with the strict proviso that no changes or edits are made and the original work is properly cited (including links to both the formal publication through the relevant DOI and the license). See: https://creativecommons.org/licenses/by-nc-nd/4.0/.

\section{References}

1. Chavan S, Bray F, Lortet-Tieulent J, et al. Platinum Priority-Review-Bladder Cancer International Variations in Bladder Cancer Incidence and Mortality. Eur Urol 2014;66:59-73.

2. Bladder cancer incidence statistics. Cancer Research UK. Available online: https://www.cancerresearchuk.org/ health-professional/cancer-statistics/statistics-by-cancertype/bladder-cancer/incidence\#ref-

3. Botteman MF, Pashos CL, Redaelli A, et al. The health economics of bladder cancer: a comprehensive review of the published literature. Pharmacoeconomics 2003;21:1315-30.

4. Edmondson AJ, Birtwistle JC, Catto JWF, et al. The patients' experience of a bladder cancer diagnosis: a systematic review of the qualitative evidence. J Cancer Surviv 2017;11:453-61.

5. Griffiths TR; Action on Bladder Cancer. Current perspectives in bladder cancer management. Int $\mathrm{J}$ Clin Pract 2013;67:435-48.

6. Zlotta AR, Van Vooren JP, Huygen K, et al. What is the optimal regimen for BCG intravesical therapy? Are six weekly instillations necessary? Eur Urol 2000;37:470-7.

7. Anastasiadis A, de Reijke TM. Best practice in the treatment of nonmuscle invasive bladder cancer. Ther Adv Urol 2012;4:13-32.

8. Mohamed NE, Gilbert F, Lee CT, et al. Pursuing quality in the application of bladder cancer quality of life research. Bladder Cancer 2016;2:139-49.

9. Blot WJ, Tarone RE. Doll and Peto's quantitative estimates of cancer risks: holding generally true for 35 years. J Natl Cancer Inst 2015;107(4):djv044.

10. Kent EE, Ambs A, Mitchell SA, et al. Health-related quality of life in older adult survivors of selected cancers: Data from the SEER-MHOS linkage. Cancer 
2015;121:758-65.

11. Li W, Qian L, He L, et al. The quality of life in patients during intravesical treatment and correlation with local symptoms. J Chemother 2014;26:165-8.

12. Abáigar-Pedraza I, Megías-Garrigós J, Sánchez-Payá J. Quality-of-life survey for patients diagnosed with non-muscle-invasive bladder cancer. Actas Urol Esp 2016;40:251-7.

13. Park J, Shin DW, Kim TH, et al. Development and validation of the Korean version of the European Organization for Research and Treatment of Cancer quality of life questionnaire for patients with non-muscle invasive bladder cancer: EORTC QLQ-NMIBC24. Cancer Res Treat 2018;50:40-9.

14. Siracusano S, Silvestri T, Bassi S, et al. Healthrelated quality of life after BCG or MMC induction for non-muscle invasive bladder cancer. Can J Urol 2018;25:9480-5.

15. Schmidt S, Francés A, Lorente Garin JA, et al. Quality of life in patients with non-muscle-invasive bladder cancer: One-year results of a multicentre prospective cohort study. Urol Oncol 2015;33:19.e7-e15.

16. Brisbane WG, Holt SK, Winters BR, et al. Nonmuscle Invasive Bladder Cancer Influences Physical Health Related Quality of Life and Urinary Incontinence. Urology 2019;125:146-53.

17. Yokomizo A, Kanimoto Y, Okamura T, et al. Randomized Controlled Study of the Efficacy, Safety and Quality of Life with Low Dose bacillus Calmette-Guérin Instillation Therapy for Nonmuscle Invasive Bladder Cancer. J Urol 2016;195:41-6.

18. Mack D, Frick J. Quality of life in patients undergoing bacille Calmette-Guérin therapy for superficial bladder cancer. Br J Urol 1996;78:369-71.

19. Abbona A, Morabito F, Rossi R, et al. Quality of life in patients undergone oncopreventive intravesical treatment for superficial bladder cancer. Arch Ital Urol Androl 2007;79:143-6.

20. Bohle A, Balck F, Wietersheim J Von, et al. The Quality of Life During Intravesical Bacillus Calmette-Guerin Therapy. J Urol 1996;155:1221-6.

21. Allareddy V, Kennedy J, West MM, et al. Quality of life in long-term survivors of bladder cancer. Cancer 2006;106:2355-62.

22. Gilbert SM, Wood DP, Dunn RL, et al. Measuring health-related quality of life outcomes in bladder cancer patients using the Bladder Cancer Index (BCI). Cancer 2007;109:1756-62.

23. Gontero P, Oderda M, Mehnert A, et al. The impact of intravesical gemcitabine and $1 / 3$ dose bacillus CalmetteGuérin instillation therapy on the quality of life in patients with nonmuscle invasive bladder cancer: Results of a prospective, randomized, phase II trial. J Urol 2013;190:857-62.

24. Koga H, Ozono S, Tsushima T, et al. Maintenance intravesical bacillus Calmette-Guérin instillation for $\mathrm{Ta}, \mathrm{T} 1$ cancer and carcinoma in situ of the bladder: Randomized controlled trial by the BCG Tokyo Strain Study Group. Int J Urol 2010;17:759-66.

25. Kowalkowski MA, Chandrashekar A, Amiel GE, et al. Examining sexual dysfunction in non-muscle-invasive bladder cancer: Results of cross-sectional mixed-methods research. Sex Med 2014;2:141-51.

26. Krajewski W, Kościelska-Kasprzak K, Rymaszewska J, et al. How different cystoscopy methods influence patient sexual satisfaction, anxiety, and depression levels: a randomized prospective trial. Qual Life Res 2017;26:625-34.

27. Sighinolfi MC, Micali S, De Stefani S, et al. Bacille Calmette-Guérin intravesical instillation and erectile function: Is there a concern? Andrologia 2007;39:51-4.

28. Singer S, Ziegler C, Schwalenberg T, et al. Quality of life in patients with muscle invasive and non-muscle invasive bladder cancer. Support Care Cancer 2013;21:1383-93.

29. van der Aa MNM, Bekker MD, van der Kwast TH, et al. Sexual function of patients under surveillance for bladder cancer. BJU Int 2009;104:35-40.

30. Yoshimura K, Utsunomiya N, Ichioka K, et al. Impact of superficial bladder cancer and transurethral resection on general health-related quality of life: An SF-36 survey. Urology 2005;65:290-4.

31. Cox E, Saramago P, Kelly J, et al. Effects of Bladder Cancer on UK Healthcare Costs and Patient HealthRelated Quality of Life: Evidence From the BOXIT Trial. Clin Genitourin Cancer 2020;18:e418-42.

32. Tan WS, Panchal A, Buckley L, et al. Radiofrequencyinduced Thermo-chemotherapy Effect Versus a Second Course of Bacillus Calmette-Guérin or Institutional Standard in Patients with Recurrence of Non-muscleinvasive Bladder Cancer Following Induction or Maintenance Bacillus Calmette-Guérin Therapy (HYMN): A Phase III, Open-label, Randomised Controlled Trial. Eur Urol 2019;75:63-71. 
33. Moher D, Liberati A, Tetzlaff J, et al. Preferred Reporting Items for Systematic Reviews and Meta-Analyses: The PRISMA Statement. PLoS Med 2009;6:e1000097.

34. Fung C, Pandya C, Guancial E, et al. Impact of bladder cancer on health related quality of life in 1,476 older Americans: A cross-sectional study. J Urol 2014;192:690-5.

35. Gilbert SM, Dunn RL, Hollenbeck BK, et al. Development and Validation of the Bladder Cancer Index: A Comprehensive, Disease Specific Measure of Health Related Quality of Life in Patients With Localized Bladder Cancer. J Urol 2010;183:1764-9.

36. Botteman MF, Pashos CL, Hauser RS, et al. Quality of life aspects of bladder cancer: A review of the literature. Qual Life Res 2003;12:675-88.

37. Gerharz EW, Månsson A, Månsson W. Quality of life in patients with bladder cancer. Urol Oncol 2005;23:201-7.

38. Wright JL, Porter MP. Quality-of-life assessment in patients with bladder cancer. Nat Clin Pract Urol 2007;4:147-54.

39. Kamat AM, Sylvester RJ, Böhle A, et al. Definitions, end points, and clinical trial designs for non-muscle-invasive bladder cancer: Recommendations from the International Bladder Cancer Group. J Clin Oncol 2016;34:1935-44.

40. Rutherford C, Patel MI, Tait MA, et al. Assessment of content validity for patient-reported outcome measures used in patients with non-muscle invasive bladder cancer: a systematic review. Support Care Cancer 2018;26:1061-76.

41. Jung A, Nielsen ME, Crandell JL, et al. Quality of Life in Non-Muscle-Invasive Bladder Cancer Survivors. Cancer Nurs 2019;42:E21-33.

42. Richards KA, Smith ND, Steinberg GD. The importance of transurethral resection of bladder tumor in the management of nonmuscle invasive bladder cancer: a systematic review of novel technologies. J Urol 2014;191:1655-64.

43. Cumberbatch MGK, Foerster B, Catto JWF, et al. Repeat Transurethral Resection in Non-muscle-invasive Bladder Cancer: A Systematic Review. Eur Urol 2018;73:925-33.

44. Mariappan P, Zachou A, Grigor KM. Detrusor Muscle in the First, Apparently Complete Transurethral Resection of Bladder Tumour Specimen Is a Surrogate Marker of Resection Quality, Predicts Risk of Early Recurrence, and Is Dependent on Operator Experience. Eur Urol 2010;57:843-9.

45. Chamie K, Ballon-Landa E, Bassett JC, et al. Quality of diagnostic staging in patients with bladder cancer: A process-outcomes link. Cancer 2015;121:379-85.

46. EAU Guidelines: Non-muscle-invasive Bladder Cancer. Uroweb. Available online: https://uroweb.org/guideline/ non-muscle-invasive-bladder-cancer/

47. Aldousari S, Kassouf W. Update on the management of non-muscle invasive bladder cancer. Can Urol Assoc J 2010;4:56-64.

48. Hendricksen K, Gleason D, Young JM, et al. Safety and Side Effects of Immediate Instillation of Apaziquone Following Transurethral Resection in Patients With Nonmuscle Invasive Bladder Cancer. J Urol 2008;180:116-20.

49. Miyazaki J, Hinotsu S, Ishizuka N, et al. Adverse Reactions Related to Treatment Compliance During BCG Maintenance Therapy for Non-muscle-invasive Bladder Cancer. Jpn J Clin Oncol 2013;43:827-34.

50. Wilson IB, Cleary PD. Linking Clinical Variables With Health-Related Quality of Life: A Conceptual Model of Patient Outcomes. JAMA 1995;273:59-65.

51. Huddart RA, Hall E, Lewis R, et al. Patient-reported Quality of Life Outcomes in Patients Treated for Muscle-invasive Bladder Cancer with Radiotherapy \pm Chemotherapy in the BC2001 Phase III Randomised Controlled Trial. Eur Urol 2020;77:260-8.

52. Blazeby JM, Hall E, Aaronson NK, et al. Validation and reliability testing of the EORTC QLQ-NMIBC24 questionnaire module to assess patient-reported outcomes in non-muscle-invasive bladder cancer. Eur Urol 2014;66:1148-56.

53. Martínez-Piñeiro L, Portillo JA, Fernández JM, et al. Maintenance Therapy with 3-month Bacillus CalmetteGuérin for 3 Years is Not Superior to Standard Induction Therapy in High-risk Non-muscle-invasive Urothelial Bladder Carcinoma: Final Results of Randomised CUETO Study 98013. Eur Urol 2015;68:256-62.

54. Ojea A, Nogueira JL, Solsona E, et al. A Multicentre, Randomised Prospective Trial Comparing Three Intravesical Adjuvant Therapies for Intermediate-Risk Superficial Bladder Cancer: Low-Dose Bacillus CalmetteGuerin (27 mg) versus Very Low-Dose Bacillus CalmetteGuerin (13.5 mg) versus Mitomycin C. Eur Urol 2007;52:1398-406.

55. Costing report: Bladder cancer Implementing the NICE guideline on bladder cancer (NG2) Putting NICE guidance into practice Costing report: Bladder cancer. Available online: www.nice.org.uk 
56. Mostafid AH, Porta N, Cresswell J, et al. CALIBER: a phase II randomized feasibility trial of chemoablation with mitomycin-C vs. surgical management in low-risk nonmuscle-invasive bladder cancer. BJU Int 2020;125:817-26.
57. Ng K, Stenzl A, Sharma A, et al. Urinary biomarkers in bladder cancer: A review of the current landscape and future directions. Urol Oncol 2021;39:41-51.
Cite this article as: Nayak A, Cresswell J, Mariappan P. Quality of life in patients undergoing surveillance for nonmuscle invasive bladder cancer-a systematic review. Transl Androl Urol 2021;10(6):2737-2749. doi: 10.21037/tau-20-1333 